\title{
XVIII.
}

\section{Ist Byzanz eine megarische Colonie?}

Daß Byzanz eine Colonie von Megara gewesen sei, wird in der neueren Geschichtschreibung als feste Thatsache angenommen. Soviel ich sehe, ist nur von éiner Seite Einsprache erhoben

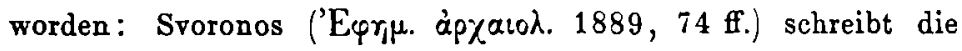
erste Gründung den Argivern zu. Er stützt sich dabei auf das Zeugnis des Hesych von Milet, der in seinen П'́xpıx. Kuvstav$\tau \iota v 0 \pi \delta \lambda \varepsilon \omega_{5} \$ 3$ (F. H. G. IV p. 147) ausdrücklich die Argiver als erste Ansiedler nennt, sowie darauf, daß die altesten Münzen von Byzanz das Bild einer Kuh auf einem Delphin, nach seiner Deutung die Io zeigen, der Iomythus aber nach Argos gehört.

Man wird sich also die Frage noch einmal überlegen müssen, um so mehr als die die Gründung betreffenden Nachrichten nirgends kritisch gesichtet sind. Wer Hesych einmal durchgelesen hat, weiß, mit welcher Vorsicht diese Quelle zu benutzen ist. Apollonius von Tyana erscheint als Zeitgenosse des Dineos, der dem Gründer der Stadt Byzas in der Herrschaft nachfolgte; unter dem dritten Herrscher greift Philipp von Macedonien die Stadt an. Was aber die Münzen betrifft, so erscheint mir die Deutung auf Io keineswegs ganz überzeugend, auch wenn wir der Versicherung Svoronos gerne glauben, daß das Thier eine Kuh, njcht wie. bisher — so auch von Head hist. num. p. 229 angenommen ward, ein Stier sei. Die gleichzeitigen Münzen der Nachbarstadt Calchedon zeigen dasselbe Thier auf einer Aehre (Aehren finden sich auch auf späteren Münzen von Byzanz); sollte die Aehnlichkeit ganz zufällig sein? Stiere und Rinder sind auf den griechischen Münzen nicht selten; die Beziehung 
des Rindes auf Viehzucht, des Delphins auf den bekanntlich zu Byzanz sehr ergiebigen Fischfang oder auf den Character der Stadt als Seestadt erscheint so einfach, daß eine mythologische Deutung zum mindesten nicht nothwendig ist. Gesetzt aber, die Deutung wäre richtig, so würden die Münzen nicht mehr beweisen, als daß Byzanz zu Ende des 5. oder Anfang des 4. Jahrhunderts - älter sind die Münzen jedenfalls nicht (s. Head p. 229, Svoronos a. a. O. p. 107) - die Localisierung der Iosage am Bosporos angenommen und auf seinen Münzen zum Ausdruck gebracht hätte. Der Versuch, den Svoronos macht, die verschiedenen Datierungen der Gründungszeit (vgl. Busolt, griech. Gesch. I ${ }^{2}$ 472, 1 und Pauly-Wissowa, Byzantion) für die Annahme einer ersten Besiedlung durch die Argiver, einer zweiten durch die Megarer zu verwerthen, kann bei dem Character dieser Gründungsdaten - vgl. Beloch, griech. Gesch. I 173 1, E. Mejer, Gesch. d. Alterth. II 443 - wenig einleuchten $\left.{ }^{1}\right)$. - Man konnte vielleicht noch anführen, daß Argos ebenfalls wie Byzanz eiserne Münzen geprägt hat (U. Köhler, athen. Mitth. VII 2). Allein diese argivischen Eisenmünzen gehören dem Anfang des 4. Jahrhunderts an, wo ein Zusammenhang zwischen Byzanz und Argos wohl jedenfalls nicht mehr bestand; die byzantinischen Fisenmünzen sind für das 5. Jahrhundert bezeugt.

Gegen Argos als Mutterstadt aber spricht schon der Umstand, daß wir von einer colonisatorischen Thătigkeit von Argos in der Mitte des 7. Jahrhunderts nichts wissen, daß Argos uberhaupt bei der sogenannten zweiten Colonisation sich nicht betheiligt hat. Die Nennung der Argiver bei Hesych ist ohne Zweifel nichts weiter als eine rationalistische Ausdeutung der Localisierung der Iosage am Bosporos.

Wie steht es aber mit dem Anspruch Megaras auf die Ehre der Gründung? Von Beziehungen zwischen Byzanz und Megarn in geschichtlicher Zeit ist nichts überliefert. Dafür, daß Calchedon eine megarische Colonie ist, haben wir das Zeugnis des Thukydides (IV 75); für Byzanz bezeugt dasselbe erst der sog. Skymnos (716 f.) Seitdem gilt allerdings Byzanz ziemlich all-

1) Man darf vielleicht annehmen, daß die Gründungszeit von $B$. rückwärts von der Einnahme der Stadt im J. 478 berechnet wurde, nach der einen Tradition auf 658 , nach der andern auf 628 , d. h. sechs bezw. fünf Generationen zu 30 Jahren vor 478 . 
gemein als megarische Colonie (Philostr. 7. soph. I 24, 3; Dionys. Byz. 14. 34 Wescher u. ofter; Joann. Lyd. de mag. III 70; Steph. Byz. s. v.; Eustath. zu Dion. Per. 803). Nun wird von O. Müller (Dorier I 120 f.), Frick (bei Pauly² II 2605;, auch noch von Busolt (gr. Gesch. $I^{2} 473,1$ ) die Uebereinstimmung in Ortsnamen, Mythen und Culten der beiden Stădte betont.

Doch sehen wir genauer zu, so hat diese Uebereinstimmung keine allzu große Beweiskraft. Was die Ortsnamen betrifft, so steht das promunturium Isthmicum nur in der lateinischen Uebersetzung des Dionysius Byz. ( $\$ 26$ ed. Frick), nicht im griechischen Text ( $\$ 32$ ed. Wescher). Wenn ein Vorsprung zu Byzanz, auf dem ein Tempel der Hera stand, "Hpa; ăxpa hieß - 80 ist wohl Dionys. B. 14 W. zu ergänzen -, so hat man kein Recht dies in Beziehung zu setzen zu dem von Strabo (VIII 380) erwähnten Heiligthum der "Hpa àxpaíx zwischen Lechaion und Pagä. Den Namen IJeprïxbs (Dion. 21 W.) vergleicht Frick mit dem Namen des Grenzgebiets zwischen Megara und Korinth, Meípaiov (Xen. hell. IV 5). Indessen erklärt sich jener Name ganz einfach als Appellativum: der jenseits des Dion. $\$ 20$ genannten Vorgebirgs gelegene Platz. Bleiben noch die

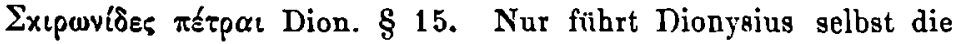
Benennung auf die Korinthier, nicht die Megarer zurilck, und auch dieser Name hat deutlich einen appellativen Character, vgl. Exıpás, Exípov, Exípos bei Pape.

Immerhin ist diese letztere Uebereinstimmung beachtenswerth. Aus den Mythen dagegen ist nichts zu gewinnen. Die Localsage vom Delphin und Karandas (Dionys. 42 W.) hat mit dem Melikertesmythus nichts zu thun; ebenso wenig darf die Localisierung der Sage von Phrixos am Bosporos (Dion. 92. $99 \mathrm{~W}$.) mit der Erwăhnung des Phrixos bei Pausanias I 44, 7, der Orts-

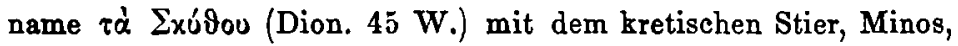
Minoa zusammengebracht werden ${ }^{2}$ ). Endlich wird die von Hesych. Miles. p. Const. 12 erwähnte Beihülfe des Apoll und Poseidon beim Mauerbau von Byzanz auf die ähnliche Sage, nach der Apoll den Alkathoos beim Mauerbau von Megara unterstützt hat (Paus. I 42, 2), bezogen. Allein, da Hesych eine sehr späte und

2) Die von Dion. gegebene Erklärung paßt nicht zu dem Namen; vielleicht ist nach Exừัou (raśpou) zu ergänzen. 
verdächtige Quelle ist, da für Megara nur Apollo, nicht auch Poseidon genannt wird, liegt es näher, an eine Entlehnung der troischen Sage zu denken.

Nicht viel günstiger steht es mit dem Verhältnis der Culte. Mehrere Heroenculte führt Dionys ohne weiteres auf die Stiftung der Magarer zurūck, die des Hipposthenes (32 W), des Schoiniklos (34), des Aias (39), des Saron (71). Nach der Ueberlieferung könnte es scheinen, als müßte man noch den Cult des Polyeidos (14) beifügen. Aber offenbar ist der Text verderbt.

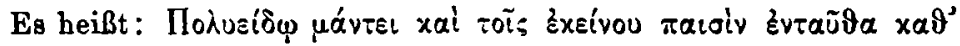

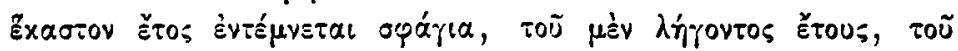

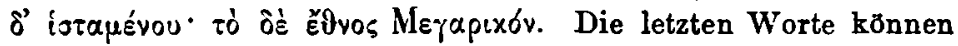
doch unmöglich die Bedeutung haben: der Cult ist von Megara hergebracht, und sie haben überhaupt keinen Zusammenhang mit dem Vorangehenden. Dagegen passen sie sehr gut an den

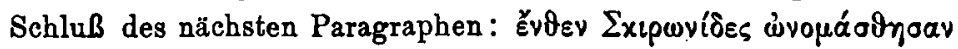

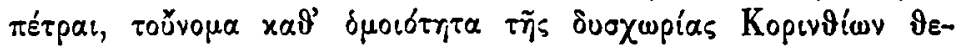

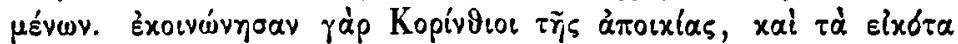

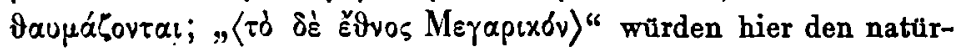
lichen Sinn ergeben: der Grundstock der Bevolkerung aber ist megarischer Herkunft. Jedenfalls würde die Erwahnung der Gräber zweier angeblicher Töchter des Polyeidos von Korinth zu Megara (Paus. I 43, 5) uns kein Recht geben, einen Cult des Polyeidos und seiner Söhne zu Megara anzunehmen. Der von Dionys genannte angebliche Wagenlenker des Amphiaraos, Schoiniklos, scheint eine sehr zweifelhafte Existenz zu sein. Es wird zwar im Lexicon des Hesych von Alexandria z. d. W. Exoívixos

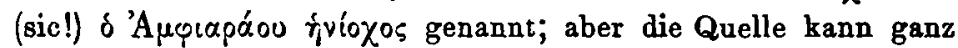
gut eben Dionys sein, und bei diesem handelt es sich wohl um nichts weiter als um eine etymologische Spielerei, zu der er oder sein Gewährsmann durch den Ortsnamen $\Sigma$ Xoivix入os bei dem dortigen Heiligthum des Amphiaraos ( $\nabla g l$. Hesych. Mil. Patr. C. 16 mit Dion. 33. 34 W.) veranlaßt wurde. Ein Heros Hipposthenes scheint sonst nicht genannt zu werden; Saron aber gehört nicht nach Megara, sondern nach Trözen (Paus. II 30, 7). Zu beachten ist auch, daß der Altar des Saron nicht in der Stadt Byzanz, sondern weiter nordwärts, am Ufer des Bosporus, stand; es ist keineswegs sicher, daß alle Heiligthümer am Bosporus auf Byzanz zurückzuführen sind. Vorüberfahrende Anwohner 
des saronischen Meerbusens mögen dem Heros Saron einen Altar gelobt und geweiht haben. Bei Aias fügt Dionys sehr gelehrt

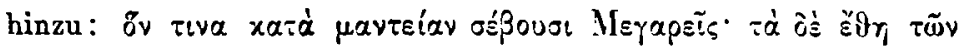

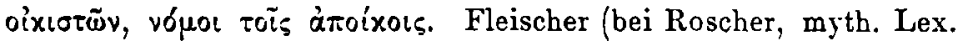
I 132) meint, Aias sei in Megara verehrt worden. Die einzige Belegstelle aber scheint eben diese Stelle des Dionys zu sein; wenn Pausanias (I 42,4) nach eigener Vermuthung das Heiligthum der Athene Aiantis auf eine Stiftung des Aias zurückführt, wenn Telamon von den megarischen Gelehrten zum Schwiegersohn des Alkathoos gemacht wurde (Töpffer att. Geneal. 271 f.), so beweist das noch nichts für die Verehrung des Aias in Megara. Man beachte auch, daß in Byzanz selbst - das von Dionys genannte Aianteion lag nordwärts am Bosporus - Achill und Aias neben einander verehrt wurden (Hesych. Mil. Patr. C. 16). Man wird also eber an Beziehungen zur troischen Küste denken müssen. Dionys hat, im Eifer zu erklären, als megarisch angesehen, was er nicht anders erklären konnte.

Es ist klar, dass eine Uebereinstimmung in den allgemeinen griechischen Culten für die Frage von keinem Belang ist. Frick vermuthet, daß Dion. $19 \mathrm{~W}$. für Zsùs áứatos $Z$ ssus à ácolos zu

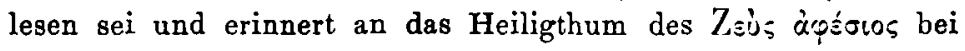
den skironischen Felsen (Paus. I 44,9). Allein die Aenderung des zweimal im lext und einmal in den Scholien bezeugten Wortes ist bedenklich, auch wenn eine befriedigende Erklärung bis jetzt noch nicht gefunden ist und die Zurückführung des Namens auf arkadische Kolonisten nicht sehr vertrauenerweckend ist und jedenfalls uns zu keiner ErkJärung verhilft.

Unter den bei Papias überlieferten byzantinischen Monatsnamen erscheint ein maleforus $=$ september, s. Bröcker und K. F. Hermann, Philol. II (1847) 248. 262 f. Bischoff Leipz. Stud. VII 374. Schon Hermann hat dabei darauf aufmerksam gemacht, daß zu Nisaia eine Demeter $\mu a \lambda o \varphi b$ pos verehrt wird (Paus. I 44, 3). Allein nāher als diese Beziehung liegt die appellative Deutung, s. Bischoff a. a. O. 376 .

Ein Typus der byzantinischen Münzen zeigt auf der Vorderseite das lorbeerbekränzte Haupt des Apollo, auf dem Revers eine gewöhnlich als Obelisk bezeichnete Săule mit Beischriften:

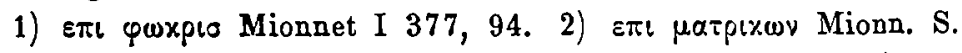
II 241, 215. 243, 229. Berlin Cat. I 147, 56 (Abb.). 3) $\varepsilon \pi \iota$ 
$\mu \varepsilon v \Xi \%$ Mionn. S. II 241, $216=$ Mus. Hunter t. XIII 24. 4) $о р x \chi \mu \alpha$ Mionn. I 377, 93. Brit. Mus. Thrace 96, 37. Aehnlich mit 3) ist 5) (zu Paris) veröffentlicht von Svoronos a. a. O. S. 92:

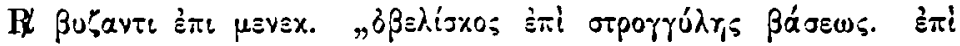

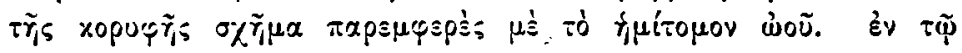

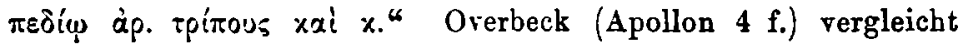
diese Säulen mit ähnlichen ${ }^{3}$ ) Figuren namentlich auf epirotischen und illyrischen Münzen (a. a. O. Münztafel I 2-8), die ohne Zweifel ein Agalma des Apollo darstellen; doch hălt er es für möglich, daß auf den byzantinischen Stücken wirklich ein Obelisk dargestellt sei, da diese Münzen erst der Kaiserzeit angehören. Der letztere Grund ist, wie Herr Prof. Dr. Pick in Gotha auf eine an ihn gerichtete Anfrage die Gũte hat mir mitzutheilen, hinfallig, das jedenfalls 1) 2) und 3), also auch 5) dem 3. oder 2. Jahrhundert vor Chr. angehören, demnach einigen der andern von Overbeck angeführten Münzen gleichzeitig sind. Derselbe Typus - „Obelisk zwischen zwei Delphinen" - erscheint nun auch auf einer megarischen Münze (Head 329), und Overbeck hat zur Erklärung die Stelle des

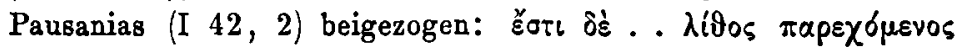

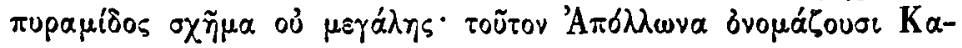
pivóv, unter der Annahme, daß die Beschreibung des Pausanias möglicherweise ungenau sei. Diese Erklärung hat Drexler (Zschr. f. Numism. XIX [1895] 128) wieder aufgenommen und die Vermuthung ausgesprochen, daß derselbe Apollo Karinos auch in Byzanz verehrt und mit jener Figur auf den Münzen dargestellt worden sei. Man sieht leicht, da $\beta$ wenn nicht andere Zeugnisse noch hinzukommen, man sich mit einer Möglichkeit wird bescheiden müssen. Eine ganz andere Erklärung giebt Svoronos;

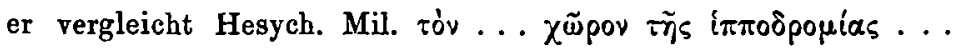

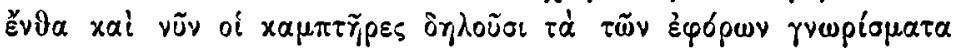

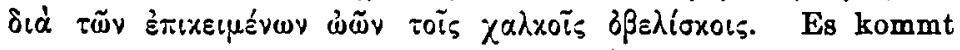
natürlich viel darauf an, ob die Figur auf dem Obelisk wirklich ein $\omega \delta \gamma$ ist; ich vermag dies aus der von Svoronos Tafel I 5 gegebenen Abbildung nicht zu erkennen; die Abbildung im Hunter'schen Katalog (oben 3) mag schlecht sein, schließt aber

3) Die Aehnlichkeit ist namentlich nach den Abb. Berlin Cat. I 147, 56 nicht zu rerkennen. 
die Deutung, die Sroronos giebt, offenbar aus. Herr Prof. Dr. Pick glaubt, daß es sich bei 1) 2) 3) wahrscheinlich um ein Agalma handle, während bei 4) an einen Obelisken gedacht werden könne (die im Kat. des Brit. Mus. gegebene Abbildung zeigt eine Saule ohne jeden Aufsatz).

Gemeinsam ist endlich den beiden Städten der Cult der Artemis b $\rho \omega \omega \sigma i ́ \alpha$, der für Byzanz aus verhältnismäßig früher Zeit (Herod. IV 87), für Megara jedoch erst aus spăterer Kaiserzeit bezeugt ist (C IG $1064=$ IGS I $113=$ Kaibel Epigr. 870). Der Beiname bpoía ist ziemlich häufig, bpowsia scheint Artemis nur in diesen beiden Städten genannt worden zu sein (vgl. PaulyWissowa II 1395). Auf die in Megara und Byzanz vorkommenden

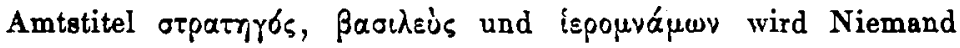
großes Gewicht legen, da sie auch sonst hăufig genug sind.

So wird das Ergebnis unsrer Untersuchung dahin zusammenzufassen zu sein, daß zwingende Belege aus Ortsnamen, Mythen und Culten für die Gründung von Byzanz durch megarische Colonisten nicht beizubringen sind. Man mag im Ernste zweifeln, ob die späteren Nachrichten dieses Inhalts nicht ein bloßer Schluß sind aus der Thatsache, daß Kalchedon megarische Colonie gewesen ist. Dieser Schluß hat aber immerhin große Wahrscheinlichkeit für sich. Der Dialect von Byzanz ist nach einem ziemlich frühen Zeugnisse (Aristoph. nub. 249) der dorische gewesen. Von dorischen Städten könnte außer Megara höchstens Korinth in Betracht kommen. Von einer Betheiligung der Korinthier bei der Gründung will auch Dionys etwas wissen ( $\$ 15 \mathrm{~W}$ ), und Genesios (p. 27 Bonn) nennt neben den Megarern Korinthier, Karystier und Mitylenäer. Außerdem stimmt die Form des byzantinischen $\beta$ mit der korinthischen überein (Kirch-

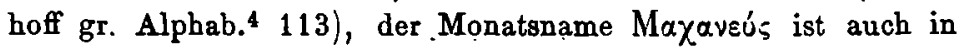
Kerkyra nachgewiesen (Bischoff a. a. O. 372). Man könnte noch an den oben besprochenen Cult des Polyeidos und seiner Söhne erinnern. Die Zeugnisse für Megara wiegen aber stärker als die hier angeführten für Korinth. Nicht unwahrscheinlich ist es, daß es sich bei Byzanz weniger als bei manchen andern Colonien $u m$ eine einmalige förmliche Gründung handelt, daß Zuzüge noch aus andern Städten und Gegenden hinzugekommen sind. Abgesehen von der Betheiligung der Korinthier ist ein boiotisches Element in Byzanz ziemlich gut bezeugt (rois 


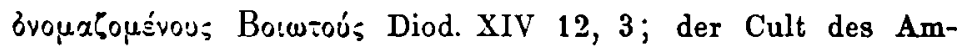
phiaraos Dion. Byz. 63, vgl. 34 ; Konstant. them. II p. 46 Bonn:

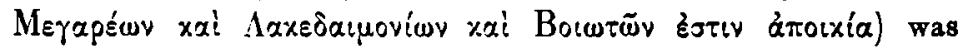
umsoweniger abzuweisen ist, als für die Betheiligung der Boioter bei der Gründung von Heraklea sichere Zeugnisse vorhanden sind (vgl. Busolt gr. Gesch. II ${ }^{2} 487$ ). Vielleicht kann man mit diesen Nachrichten die Notiz des Aristoteles (pol. 1303 a 33) von den Ě זoเพ०:, die den ursprünglichen Ansiedlern gegenüber gestanden haben, zusammennehmen; doch handelt es sich hier wahrscheinlich um später zugewanderte. Völlig werthlos für unsere Frage sind dagegen die Angaben, die auf bloßer Ausdeutung ron Ortsnamen am Bosporos beruhen (Dion. Byz. 47; Hesych. 20, Erwähnung der Rhodier; Dion. 48 der Thasier, 81 der Lykier).

Stuttgart.

J. Miller.

\section{Die Besiedlung Nordafrikas nach Sallust, Jug. 18.}

Wie kommen Meder, Perser und Armenier dazu, einen Bestandtheil des "Heeres des Herkules" zu bilden? Die Versuche der Früheren, einen wirklichen Zusammenhang zwischen den afrikanischen und jenen asiatischen Volkern nachzuweisen, wird heute Niemand wiederholen. Sallust selbst weist uns einen Weg zur Erklărung: $\S 10$ nomen eorum paulatim Libyes corrupere, barbara lingua Mauros pro Medis adpellantes. Nur kann Sallust's bezw. des 17, 7 genannten Hiempsal Gewăhrsmann, vermuthlich ein findiger Grieche, nicht von der kühnen Gleichung Mauri = Medi ausgegangen sein. Dagegen lag es nahe, die Pharusii, wie Plin. h. n. 5, 46 mit deutlicher Beziehung auf unsere Stelle thut, mit den Persae zu identificieren, oder noch eher die offer genannten Perorsi, die vielleicht mit den Pharusii identisch sind, und deren Wohnsitze denen der Persae bei Sallust entsprechen. Bei den Armenii kann man entweder an die Ptolem. IV 6, 21 genannten 'Appial oder an den Fluß Armua in Numidien (Plin. $\mathrm{V}$ 16), oder - die gröbste Etymologie hat vielleicht die meiste Wahrscheinlichkeit - an die Garamanten denken. Man suchte also an den Namen der afrikanischen Völkerschaften einen Anhaltspunkt, um die Besiedlung Nordafrikas zu erzăhlen; einen solchen Anhaltspunkt boten die Perorser - Perser; nach den Persern wurden auch Armenier und Meder entdeckt, und als Führex dieser Völker fand sich kein geeigneterer Mann als Herakles.
Stuttgart.
J. Miller. 